\title{
Effect of Non-uniform Distribution of Electric Field on Diffused Quantum Well Lasers
}

\author{
W.M. Man \& S.F. Yu \\ Department of Electrical \& Electronic Engineering, The University of Hong Kong, \\ Pokfulam Road, Hong Kong \\ E-mail: wmman@hkueee.hku.hk
}

\begin{abstract}
The influence of non-uniform distribution of electric field on the steady state and dynamic behavior of diffused quantum well lasers is studied by a selfconsistent model. The energy band structure of the quantum well can be distorted significantly by the non-uniform distribution of electric field, especially for devices with diffused quantum well structure. Hence, the evaluation on the optical gain as well as the modulation responses of diffused quantum well lasers using flat band approximation can be deviated significantly with nonuniform distribution of electric field taken into consideration.
\end{abstract}

\section{Introduction}

Recently, many theoretical models have been established to investigate the optical properties of semiconductor lasers with diffused quantum well (QW) structures [1],[2]. In their studies, the optical gain and spontaneous emission rate of diffused QW are calculated under the assumption of symmetric flat band approximation. However, detailed investigation has shown that the energy band structure of QW can be distorted at high forward bias voltage as the non-uniform distribution of electric field (arisen from the accumulation of charges near the interfaces of the well and barriers) [3] has modified the optical gain and spontaneous emission rate of the QW [4]. This is because the variation of carrier confinement inside the $\mathrm{QW}$ alters the effective mass as well as the strain of the QW. Furthermore, interdiffusion increases the well width and the amount of charges stored inside the $\mathrm{QW}$; the distortion on energy band of QW can be larger than that of the as-growth one. This implies that the calculation of optical gain and spontaneous emission rate of devices with diffused QW using symmetric flat band approximation is not appropriate and a self-consistent treatment with non-uniform distribution of electric field and charges taken into consideration is required.

In this paper, a simplified self-consistent model for QW lasers is developed with the continuity of quasi Fermi potentials adopted for the calculation of quasi 2D confined carrier distribution inside the well.

\section{Device Structure and Modeling Procedures \\ A. Laser Structure and Material Parameters}

Separate confinement heterostructure $(\mathrm{SCH})$ of a single QW laser is formed by sandwiching the InGaAs/GaAs QW between the $\mathrm{p}-\mathrm{AlGaAs}$ and $\mathrm{n}$-AlGaAs cladding layers. The non-uniform distribution of material composition of the p-i-n structure can be characterized by the position-dependent material parameters such as the semiconductor permittivity, the low-field mobilities, the energy bandgap, the electron affinity and the effective density of states for the semiconductors.

\section{B. Laser Model}

The detailed dynamic model for a SCH QW laser under carrier injection can be described by a set of coupled equations.

\section{1) Electrical Model}


Poisson's equation for electrostatic potential $\mathrm{V}$, and the time dependent current continuity equations for electrons and holes.

$$
\begin{aligned}
& \frac{\partial}{\partial \mathrm{x}}\left(\varepsilon \frac{\partial \mathrm{V}}{\partial \mathrm{x}}\right)=-\mathrm{q}\left(\mathrm{N}_{\mathrm{D}}-\mathrm{N}_{\mathrm{A}}+\mathrm{p}-\mathrm{n}\right)(1) \\
& \frac{\partial \mathrm{n}}{\partial \mathrm{t}}=\frac{1}{\mathrm{q}} \frac{\partial \mathrm{J}_{\mathrm{n}}}{\partial \mathrm{x}}-\mathrm{R} \\
& \frac{\partial \mathrm{p}}{\partial \mathrm{t}}=-\frac{1}{\mathrm{q}} \frac{\partial \mathrm{J}_{\mathrm{p}}}{\partial \mathrm{x}}-\mathrm{R}
\end{aligned}
$$

where $\varepsilon$ is the static dielectric constant, $\mathrm{V}$ is the electrostatic potential, $q$ is the magnitude of electron charge and $R$ is the recombination rate of carriers. $n$ and $p$ denote the electron and hole concentrations, respectively. The electron current density, $J_{\mathrm{n}}$, and hole current density, $J_{p}$ in (2) and (3), due to drift and diffusion, are given by

$$
\begin{aligned}
& J_{n}=q D_{n} \frac{\partial n}{\partial x}-q \mu_{n} n \frac{\partial E_{F n}}{\partial x} \\
& J_{p}=-q D_{p} \frac{\partial p}{\partial x}-q \mu_{p} p \frac{\partial E_{F p}}{\partial x}
\end{aligned}
$$

where $\mu_{\mathrm{n}}$ and $\mu_{\mathrm{p}}$ denote the carrier mobilities for electrons and holes, and $D_{n}\left(=k_{B} T \mu_{n} / q\right)$ and $D_{p}\left(=k_{B} T \mu_{p} / q\right)$ are the electron and hole diffusion coefficients, respectively. $T$ is the temperature in Kelvin. $\mathrm{E}_{\mathrm{Fn}}$ and $\mathrm{E}_{\mathrm{Fp}}$ are the Fermi energies of electron and hole, respectively.

\section{2) Optical Model}

The scalar wave equation is solved to obtain the transverse optical field, $\widetilde{E}(x)$, and the propagation constant $\beta$. The scalar wave equation is given by

$$
\left[\frac{\partial^{2}}{\partial \mathrm{x}^{2}}+\left(\varepsilon(\mathrm{x}) \mathrm{k}_{\mathrm{o}}^{2}-\beta^{2}\right)\right] \widetilde{\mathrm{E}}(\mathrm{x})=0
$$

where $\mathrm{k}_{0}\left(=2 \pi / \lambda_{0}\right)$ is the wavevector in vacuum and $\lambda_{0}$ is the lasing wavelength. The electric permittivity, $\varepsilon$, dependent on the material composition and carrier densities, which can be written as

$$
\varepsilon(x)=\varepsilon_{r}(x)-\alpha_{f 1} \frac{\sqrt{\varepsilon_{r}(x)}}{k_{o}} g(n, p)+i \frac{\sqrt{\varepsilon_{r}(x)}}{k_{o}}(g(n, p)-\alpha)(7)
$$

where $\varepsilon_{\mathrm{r}}$ is the background relative permittivity, $\alpha_{H}$ is the linewidth enhancement factor and $\alpha$ is the free carrier and scattering loss coefficient.

The photon density, S, inside the laser cavity can be determined by the photon rate equation:

$$
\frac{\partial \mathrm{S}}{\partial \mathrm{t}}=\mathrm{v}_{\mathrm{g}}\left(\mathrm{G}-\alpha_{\mathrm{eff}}\right) \cdot \mathrm{S}+\overline{\mathrm{R}}_{\mathrm{sp}}
$$

where $\mathrm{G}$ is the effective optical gain, $\alpha_{\mathrm{eff}}$ the effective cavity loss, $\overline{\mathrm{R}}_{\mathrm{sp}}$ the spontaneous-emission recombination rate.

\section{3) Numerical Technique}

The laser is divided into finite sections along the transverse direction. The set of coupled differential equations is linearized using finite difference method on the onedimensional grid division point [5],[6]. The transient response of the QW laser can be calculated self-consistently by using Newton's method.

\section{Numerical Results}

Figure 1 shows the distribution of electric field inside the as-growth and interdiffused QW laser. The change of the electric field is caused by the distortion of confinement profile of the QW from its original semirectangular shape. The distortion of $\mathrm{QW}$ shape is due to the out diffusion of indium from the well to the barrier such that the indium fraction is reduced in the well.

In our analysis, the extent of interdiffusion is characterized by a diffusion length, $\mathrm{L}_{\mathrm{d}}$, where $\mathrm{L}_{d}=0 \AA$ represents the as-growth QW. As a result, the distribution of wavefunction and the sub-band energies are changing subject to the degree of the disordered potential of the QW.

In the following analysis, the transient response of lasers with as-growth and diffused (of $\mathrm{L}_{\mathrm{d}}=10 \AA$ ) QW are investigated under step voltage modulation. For the diffused QW of $L_{d}=10 \AA$.

Figure 2 shows the transient response of photon density, S, and effective optical gain, 
$\mathrm{G}$, of laser with as-growth QW. The laser is initially biased at their threshold voltage $\left(\mathrm{V}_{\mathrm{th}} \approx 1.20 \mathrm{~V}\right)$ and modulated with a voltage step of $0.078 \mathrm{~V}$ such that the steady state photon density at the facets is around $3 \times 10^{15} \mathrm{~cm}^{-3}$. The solid and dotted lines are the calculations using constant flatband approximation and the time variation of energy band and electric field, respectively. It is observed that the transient responses of $\mathrm{S}$ as well as $\mathrm{G}$ with and without constant flat band approximation exhibit same output results.

Figure 3 shows the transient response of $\mathrm{S}$ and $\mathrm{G}$ of laser with diffused $\mathrm{QW}$ of $\mathrm{L}_{\mathrm{d}}=$ $10 \AA$. The laser is initially biased at their threshold voltage $\left(\mathrm{V}_{\mathrm{th}} \approx 1.20 \mathrm{~V}\right)$ and modulated with a voltage step of $0.082 \mathrm{~V}$. In this case, photon density is underestimated using constant flat band approximation. This is because the effective optical gain is underestimated as shown in figure $3 b$.

The variation of optical gain due to large forward bias voltage can be explained as follows: In the flat band approximation, the variation of electric field distribution inside the $\mathrm{QW}$ is ignored in the calculation, however, rapid change in electric field inside the QW (see figure 1) causes the accumulation of positive charge inside the interfaces of the QW but negative charge just outside the QW such that the energy band diagram of QW is further distorted. Therefore, the flat band approximation will overestimate the optical gain and the output photon density of the laser. In addition, the variation of electric field for $L_{d}=10 \AA$ is larger than that for $L_{d}=0 \AA$.

\section{Conclusion}

A simplified self-consistent model is developed, using the assumption of quasiFermi potentials, for the calculation of modulation responses of diffused QW lasers. It is shown that the optical gain of devices with diffused QW structure can be altered by the distortion of energy band diagram due to the non-uniform distribution of carriers and electric field such that the symmetric flat band approximation may not be suitable for the application in devices with diffused QW structure.

\section{References}

[1] S.F. Yu, C.W. Lo \& E.H.Li,'Investigation of high power single mode operation in DFB and FP lasers using diffused quantum wells structure', IEEE J. Quantum Electron., Vol.33, no.6, pp.9991009, 1997.

[2] S.F.Yu \& W.M. Man,'Comprehensive modeling of diffused quantum wells vertical cavity surface emitting lasers', submitted to IEEE J. Selected Topics in Quantum Electron. fall 1998 issue for publication.

[3] J. Lee, M.O. Vassell and G.J.

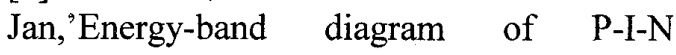
heterostructures for single quantum well lasers', IEEE J. Quantum Electron., vol. 29, no.6, pp. 1469-1475, 1993.

[4] P.A. Chen, C. Juang and C.Y. Chang,'Carrier induced energy shift in GaAs/AlGaAs multiple quantum well laser diodes', , IEEE J. Quantum Electron., Vol. 29, no. 10, pp. 2607-2618, 1993.

[5] M. Kurate, "Numerical analysis for semiconductor devices", Lexington: Lexington Books, 1982.

[6] D.L. Scharfetter and H.K. Gummel, 'Large-signal analysis of a silicon read diode oscillator', IEEE Trans. Electron Devices, vol. ED-16, p.64-77, 1969. 

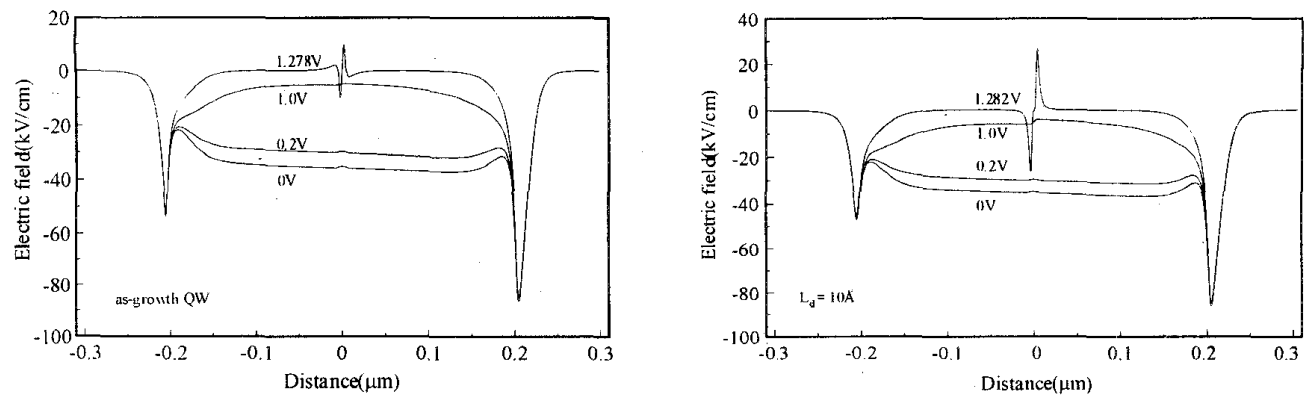

Figure 1 Distribution of electrical field of SCH with (a) as-growth $\mathrm{QW}$ and (b) diffused $\mathrm{QW}$ with $\mathrm{L}_{\mathrm{d}}=10 \AA$ at different $\mathrm{V}_{\mathrm{a}}$ as indicated in the diagrams.
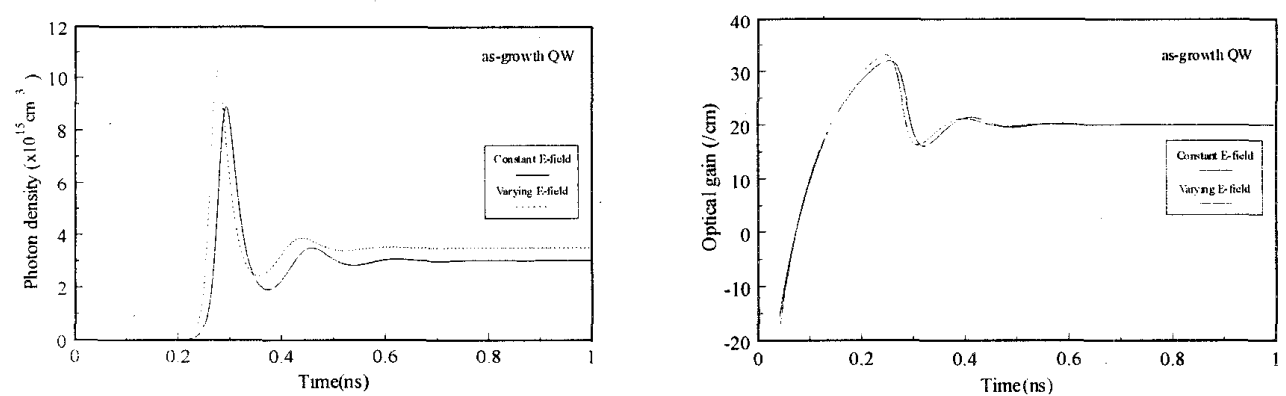

Figure 2 The transient response of (a) optical power and (b) effective optical gain of SCH-QW laser with asgrowth QW. Solid lines are for constant electric field and dotted lines are for variation of electric field.
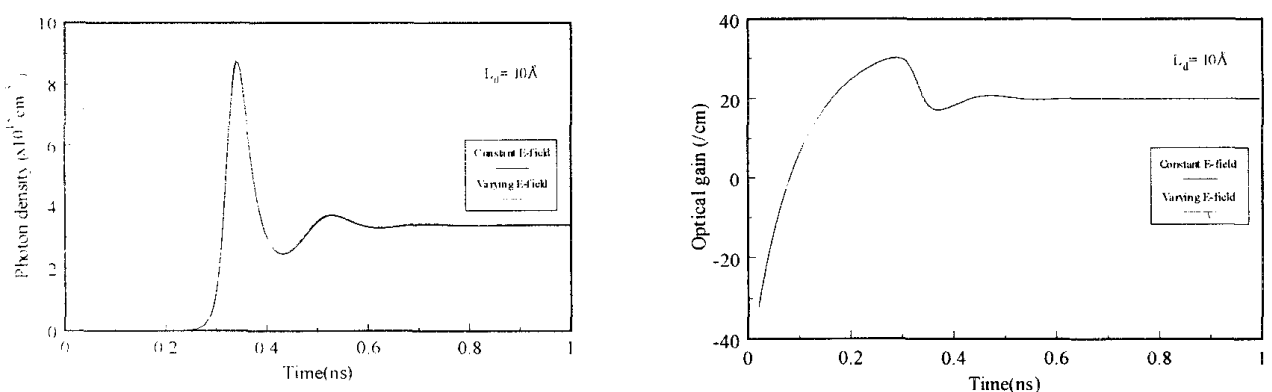

Figure 3 The transient response of (a) optical power and (b) effective optical gain of SCH-QW laser with diffused $Q W$ of $L_{i}=10 \AA$. Solid lines are for constant electric field and dotted lines are for variation of electric field. 